\title{
Study Recruitment Status
}

National Cancer Institute

\section{Source}

National Cancer Institute. Study Recruitment Status. NCI Thesaurus. Code C93480.

Status of finding and enrolling appropriate study subjects (those selected on the basis of the protocol's inclusion/exclusion criteria) into a study. 